\title{
Visualization Cockpit: Orchestration of Multiple Visualizations for Knowledge-Exploration
}

\author{
$\underline{\text { doi:10.3991/ijac.v3i4.1473 }}$ \\ K. Nazemi, M. Breyer, D. Burkhardt, D. W. Fellner \\ Fraunhofer Institute for Computer Graphics Research, Darmstadt, Germany
}

\begin{abstract}
Semantic-Web technologies and ontology -based information proc essing sy stems ar e e stablished te chniques, in more th an only research areas and institutions. Different worldwide $p$ rojects an $d$ en terprise companies id entified already the adde d value of $s$ emantic tec hnologies, so the $y$ work on diffe rent sub-to pics for gathe ring and $c$ onveying knowledge. As the p rocess of gathering and structuring semantic information plays a key role in the most de veloped applications, the pr ocess of transferring and adopting knowledge to and by human $s$ is ne glected, although the complex $s$ tructure of $k$ nowledge-design op ens man y research-questions. Th e cu stomization of th e p resentation itself and the interaction techniques with these presentation artifacts is a ke $y$ que stion for gainful and e ffective work with semantic information. The following paper describes a new approach for $v$ isualizing semantic information as a composition of different adaptable ontology -visualization techniques. We start with a categorized description of existing ontology visualiz ation techniques and show potential gaps.
\end{abstract}

Index Terms-Exploratory Learning, Intelligent User Interfaces, Semantic Visualization, Semantic Web.

\section{INTRODUCTION}

Semantic-Web and ontology-based information systems play more and more a key-role in today's information processing systems. Not only research institutions have recognized the added-value of these technologies, there are many enterprise and non-profit institutions using ontologies for structuring, transferring and adopting knowledge. Ontologies have become an established data model for conceptualizing knowledge entities and describing semantic relationships between knowledge entities and domains. They are used to model the concepts of specific domains and are widespread in the areas of the semantic web, digital libraries and multimedia database management [13]. This makes them a powerful technology for information management and decision support systems. However, these ontologies may become very large and complex what makes it difficult for the average user to understand the underlying knowledge space [15]. Likewise with these average users a domain experts should not be burdened with the need to learn the complex range of functions ontologies offer. The surplus value of this information structure should be utilized in a way the user can explore and acquire the knowledge. To alleviate ontology exploration and knowledge acquirement, visualizations are needed, so that users are able to gain the most benefit.

Different ontology visualization techniques consider different aspects of ontologies or focus on various but specific ontology characteristics, e.g. displaying the hierarchical inheritance structure, multiple inheritance and semantic relations between ontology entities [13]. The visualization of the complex structure of ontologies with concepts, individuals and relations as fundamental basis, tends to result in visualization with reams of graphs, lines and icons. For this reason is the usage of a single visualization not adequate for all tasks or all users. It is necessary to combine different visualization techniques and reduce the complexity of information by splitting in different separated areas of ontology visualization. Further the different tasks and users have diverse requirements. Users e.g. different precognitions and previous knowledge, where one user is overstrained with same visualization another user can be under challenged.

In this paper a new approach of ontology visualization technique by composing different visualization methods in a user-adaptable way will be described. Every user has the ability to choose within a "pool" of visualizations a number of adequate visualization and combine them as knowledge cockpit. Further he is able to choose colors, icons relation-types etc. for a better comprehension of the knowledge domain.

The paper describes different existing visualization techniques using a classification of Knowledge Visualizations for semantically annotated information. By classifying existing visualization methods the adoption of the visualization in different usage scenarios, e.g. exploratory learning or searching will be discussed. This classification will allow identifying adequate visualization techniques for a given learning scenario.

After that a new approach of a knowledge cockpit by graphical visualization of ontologies will be presented, which allows visualizing the formal description of knowledge as onologies in different way by using a single User Interface. The paper will depict that Knowledge exploration is important for adopting knowledge with information system, whereas graphical representation of the knowledge can help to optimize the learning process and reduce the cognitive overload.

\section{RELATED WORK}

Nowadays there are many different approaches for visualizing ontologies. In this section we present an overview of different ontology visualization techniques and discuss the advantages and drawbacks. We start with a short technical introduction to the fundamentals of ontologies and their structure and continue with describe a selected set of visualization techniques representing the most common and valuable visualizations. 


\section{A. Semantic and Ontology: A fundamental description}

For ontology visualization the three most important data elements are concepts, individuals and relations. These elements can contain further properties which describe various features and attributes. Concepts of an ontology represent abstract models of entities in the domain of interest [11]. Concepts are defined as terminological statements in the schema, which tends to be more permanent. A concept can inherit properties from other concepts using the subclass-of relation. This inheritance structure constitutes an overlapping hierarchy describing the domain of interest as generalized concepts which become more and more specific downwards this hierarchy. Next to this inheritance structure semantic relations are defined in the schema to model contextual references.

On the instance level of the ontology individuals are defined which instantiate concepts and specify inherited properties. The individuals represent real world objects and are the actual data of the modeled domain. Furthermore semantic relations between individuals are specified on the instance level to model a concrete relationship between two individuals. Each relation has a direction, a type and a label. Especially this label is important for the field of ontology visualization, so the user can more easily understand the semantics of that relationship [28].

To ensure a reasonable schema-design ontology-experts work on the process of conceptualizing the domain of interest. But besides the design for reliable reasoning, ontologies are designated to be used as databases for applications domain-experts and common users interact with. To gain the most benefit for the common users an ontology visualization technique must be able to impart the multiple inheritance, the concept hierarchy and the semantic relations between ontology entities [13].

\section{B. Existing Ontology Visualization Techniques}

Existing ontology visualization for imparting knowledge can be categorized in following techniques: Intented Lists, Node-Link Visualizations, Zoomable Visualization, Space-Filling Visualization and Context, Focus and Distortion Visualizations [13].

Indented Lists are tree-based visualizations that offer a Windows Explorer like tree view of an ontology. Because of their familiarity to the common user, indented lists are easy to use and allow high performance in ontology exploring [13]. They are used in most of the ontology management systems like the Protégé Class Browser [18], OntoRama [7] and Kaon Tree-based visualizations provide a clear view of entity labels and the concept hierarchy. However, this kind of visualization has several drawbacks in the task of ontology visualization. Indented lists are only applicable for representing the hierarchical part of the ontology. Thus the representation of semantic relations and multiple inheritance is not feasible. Furthermore, only a limited part of the ontology can be displayed at once. The top-down layout results in poor space-filling causing the need for scrolling during ontology exploration [20]. For this reason indented lists are not very applicable for imparting the general structure of the ontology.

Node-link visualizations represent the concepts and individuals as nodes and relations as edges. In contrast to indented lists, the representation of multiple inheritance and semantic relations is feasible, by interconnecting a child with edges to all its parents. For this reason this visualization technique is used for many different ontology visualizations, like OntoViz [26], IsaViz [21], OntoTrack [16], OntoSphere [5] and WSMOViz [14]. Nodelink visualizations are well suited for imparting an overview of the entire ontology structure. Nevertheless they make inefficient use of screen space [22]. For large datasets this leads to an insufficient presentation of the whole structure of the ontology what results in context-loss and the need for scrolling. Also the visualization of many relations may result in confusing diagrams with overlapping edge labels.

In Zoomable Visualizations the hierarchy of the ontology is represented by nesting nodes of lower levels inside their parents. Usually the user is able to zoom into child nodes to gather information from items at deeper levels. This visualization technique is used for the visualization plug-in Jambalaya [27] for the Protégé ontology tool, CropCircles [20] and SemaSpace [4]. Zoomable ontology visualizations provide a clear overview of the ontology's hierarchy. The user is able to request details-on-demand for items of interest by zooming into the desired entity which reduces the cognitive load of the user. But on the other hand the context of the selected element is lost and in some cases it is difficult to recognize the parent node of the zoomed entity or to identify its level in the hierarchy. Relations between the ontology elements are usually visualized as directed, labeled links and are displayed by default (Jambalaya) or on-demand (CropCircles). If the ontology contains many relations this type of relation visualization ends in visual clutter and overlapping labels what makes it difficult for the user to acquire the needed information.

Space-filling visualizations are based on the concept of using the whole screen space by subdividing the available space for a node among its children [13]. The best known representative of space-filling visualizations is the Treemap visualization proposed from Shneiderman [25]. It uses a 2D approach of space-filling to represent hierarchies, in which each node is a rectangle and has been applied by Baehrecke et al. for visualizing ontologies [3]. Treemaps are efficient when users are interested in the leaf nodes and provide a good overview if the hierarchy is trivial [13]. If the hierarchy becomes larger and deeper, significant cognitive effort is needed to understand the hierarchical structure of the visualized information [31], so this type of visualization does not offer an efficient way to impart knowledge from complex ontologies [17]. Another space-filling approach for visualizing ontologies is SeMap, proposed from Nazemi et al. [17]. SeMap allows the incremental exploring of the ontology's hierarchy what reduces the cognitive load of the user. The exploration starts with the root node and the user can expand a single path of entities of interest. The main drawback of both visualizations is that they are only applicable for visualizing the hierarchy of ontologies and are not feasible for an appropriate visualization of semantic relations and multiple inheritance.

Context, Focus and Distortion Visualizations are based on the concept of distorting the view of a visualized graph. The user is able to select a node of interest in order to focus and enlarge it. The focused node is usually centered and other nodes are placed around the focused node reduced in size. This technique is used in ontology visualizations like TGVizTab [1] and OntoRama [7]. Both representatives are based on graph visualizations and thus 
they are able to represent multiple inheritance and relations between the entities of the ontology. The advantage of context, focus and distortion visualizations is that an entity of interest is visualized without losing its context. The drawback of this visualization technique is that the position of nodes alters when the user selects a new node and thus it is complicated for the user to keep track of the visualized ontology structure and to understand the complete inheritance structure.

\section{COMPOSITION OF ONTOLOGY-VISUALIZATIONS TO EXPLORE AND ADOPT KNOWLEDGE}

\section{A. Knowledge Exploration using Semantic Data Structures}

Ontology is a formal, machine readable description of knowledge, whereas today's learning processes are not formal anymore. A common scenario of gathering and adopting knowledge on web could be the usage of Wikipedia. The user starts searching for a specific topic and finds an article on Wikipedia. The most users read the main description and scroll down to the different headlines of interest. But the process of knowledge exploration has just begun now. The users find in the searched article hyperlinks to other topics and want to know more. As we made a small evaluation with students, who had to answer a very simple question using Wikipedia, we could observe nearly all students read articles related to the main one and spend more time with the related articles, a typical behavior of exploring knowledge.

Semantically annotated knowledge has more associations between the knowledge entities and is further explicit and entitled. The users have the possibility to navigate through the relations of the knowledge assets and learn while interacting. The knowledge exploration can be more structured using the meaningful relations between the learning objects of interest, and so improve the process of knowledge acquisition.

\section{B. Visualization Techniques for Knowledge Exploration}

Knowledge exploration is an important process for adopting knowledge with information system, whereas graphical representation of the knowledge can help to optimize the learning process and reduce the cognitive overload. As we described in our related work section, different ontology visualization techniques try to solve the problem and offer an adequate way to visualize ontology in different ways for different tasks and users. But further we showed that the visualizations have drawbacks or disadvantages in usage or interaction. To provide an added value for learning with graphical systems, the visualization should be usable without an additional learning expense. The main criteria of a visualization should be the reduction of the usage complexity and hence also the learning expense. How is it possible to reduce the complexity of a system without loosing main functionalities or main information?

A knowledge visualization cockpit breaks the complexity of a single visualization down into several visualizations. Each of the cockpit visualizations is easy in its way of interaction and knowledge acquisition. And with the orchestration of the visualizations the complex information can be visualized without complex visualizations. In the following section this visualization cockpit will be introduced, beginning with a description of some ontology visualization techniques, which will be further composed to a knowledge cockpit. First of all it is necessary to describe the functionalities and how they complement each other:

\section{SEMAP}

SeMap is a combination of the Shneiderman's Treemap and Treeview [17]. The Semantic Map (SeMap) uses the two graphical metaphors, Treemap and Treeview, to combine the surpluses for a special case: the usage of semantic annotated data and the implicit impartation of knowledge.

Figure 1 shows a screenshot of seMap.

Graphical primitives like color, order and size are used to communicate relevant information in a way the user can fast and proper percept it. Color indicates user specific relevance whereas the order and size are determined by a combination of user- and data-based relevance. Order arranges the most relevant element next to the selected element of the last row, as the arrangement of the blue elements in figure 1 indicate.

SeMap is an ontology visualization that only visualizes the concepts and their hierarchy. It is possible to navigate through the set of concepts, where different graphical primitives indicate the relevance of the concept. The navigation is very fast, the information is highly reduced for an abstracted perception.

\section{SEMASPACE}

SemaSpace is a visualization of knowledge spaces, modeled as ontologies (concepts, instances, relationships etc.), supporting different aspects, e.g. thematic, cooccurrences, spatial, clusters, or configurable domainspecific representations. It provides different knowledge domains (ontology concepts) visualized as circles containing the instantiation of the knowledge domain as smaller circle. The semantic visualization tool SemaSpace offers a sophisticated way to explore knowledge spaces. It offers concepts and related knowledge items to them as factual knowledge and interrelation between knowledge spaces. Awareness knowledge is acquired, when the user explores knowledge spaces and makes decisions to follow different branches or chooses alternative branches in the semantic visualization. The interactive navigation of SemaVis allows users to explore knowledge spaces, to filter different aspects, to follow different branches, or return to the starting point. Users can also

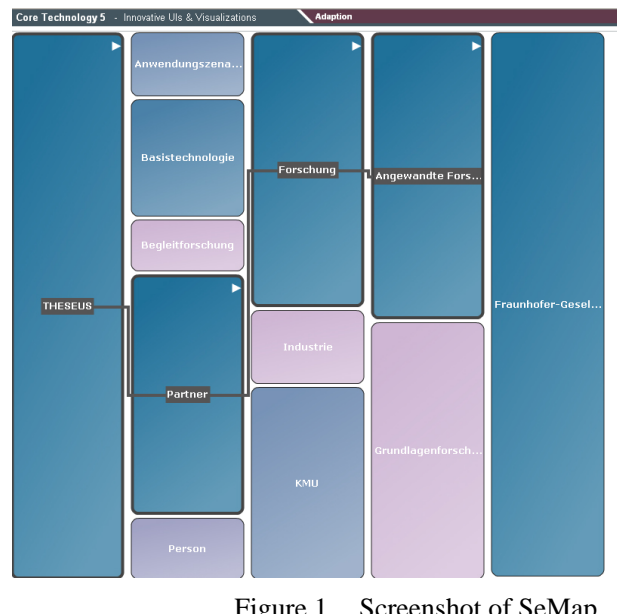

Figure 1. Screenshot of SeMap 
reorganize the semantic visualization of knowledge spaces to put the important or relative knowledge spaces in the focus just like working on the desktop. It means that users have a very active role in exploring knowledge spaces, which leads to gain active experience about knowledge spaces instead of just passive learning. [4]

The following figure shows a screenshot of the SemaSpace visualization:

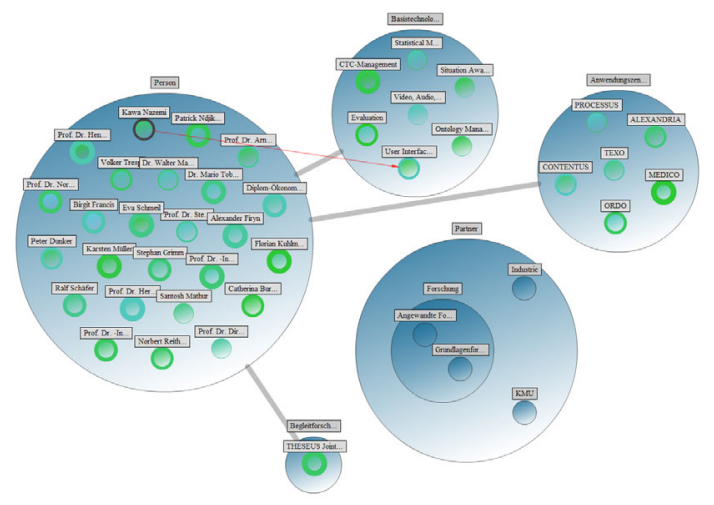

Figure 2. SemaSpace

The SemaSpace visualization contains the most information about a set of concepts within an ontology. It visualizes concepts, relations between individuals and the individuals themselves. The user has a full information overview of the given ontology, but the information could be very complex for some users or if many entities should be visualized.

\section{SEMAGRAPH}

The SemaGraph Visualization is a very simple visualization that provides different graphical algorithms for visualizing individuals and their relations. The user is able to navigate through a set of individuals with the first grade relations.

The following figure shows a screenshot of various SemaGraph implementations:

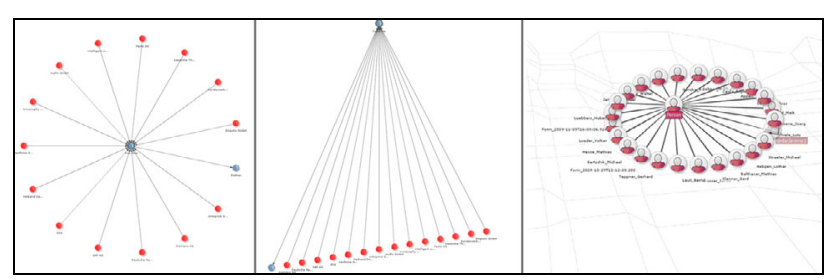

Figure 3. SemaGraph Derivations

The SemaGraph visualization provides an adequate navigation through all individuals of a concept and offers a very simple to use user interface. The information details are reduced on individuals and their first grade relations.

All the described visualizations have their own advantages and disadvantages. SemaSpace visualizes all information, but is too complex to understand and to use, SemaGraph and SeMap are very easy to use, but do not offer all the required information. It is necessary to provide a visualization that covers all aspects and is adaptable enough to create a personalized user interface.

\section{SEMASUN}

SemaSun is a visualization that uses the sunburst metaphor for presenting structured data. Our approach of the SemaSun visualization follows the Visual Information-
Seeking Mantra proposed by Shneiderman. First the user is able to gather an overview of the entire ontology to understand the complete structure of the knowledge space. After gaining an overview by exploring the inheritance structure, the user is able to focus on entities of interest and to request details of these entities on demand. The exploration in such visualization starts in the middle from a very abstract point of view, to a concrete view on the borders of the sunburst visualization. So the user is able to easily explore the data source and to understand the knowledge space. [35]

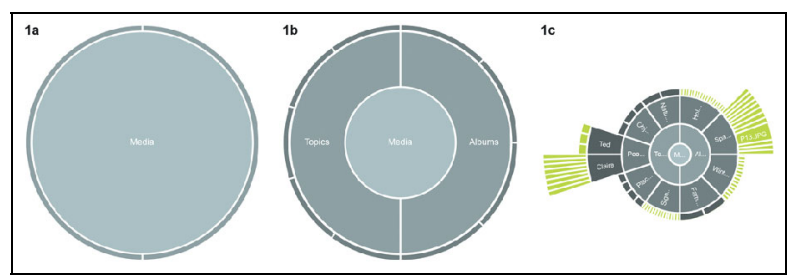

Figure 4. Incremental semantics data exploration without losing contextual information.

To reduce the cognitive overload of the user, the SemaSun visualization does not show the entire structure of the dataset as initial state. On start-up of the visualization, the root concept of the ontology is shown (Figure 4a) and the user is able to incrementally explore the hierarchy of the ontology by expanding entities of interest (Figure 4b). The radial layout of the sunburst visualization offers thereby the expansion of multiple paths (Figure 4c) so users are able to gather an overview of the whole inheritance structure and are not limited to the exploration of a single path. To maintain the informational context and to avoid the need for scrolling, entities that users visited earlier move closer to the center and are reduce in their size If the user moves the mouse over an entity whose label is not completely visible the size and the angle of the entity is adjusted so that the whole label is visible. This distortion technique is especially important if the slices become thin or the user wants to explore small elements near the center. To advise users of the existence of child nodes, an expandable mark is shown around entities that are expandable. The number of child nodes is denoted as the number of arcs around the parent node.

\section{SEMATIME}

Time appears in many different domains as a common attribute, like in digital libraries, criminal investigation and medical information systems to support users in understanding chronological structures. SemaTime is an interactive timeline visualization especially designed for depicting time-dependent semantics. [36]

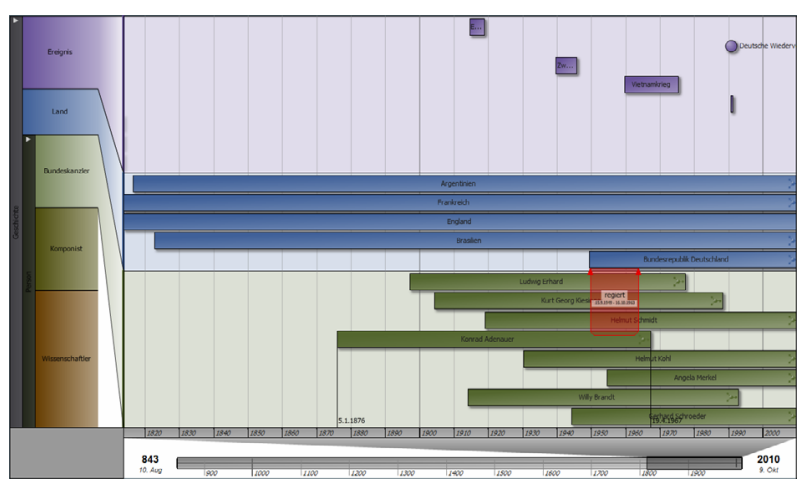

Figure 5. SemaTime - time dependent data visualization 
In principle SemaTime is analogous designed as a typical coordinate system. On the horizontal axis is the temporal dimension while on the vertical axis the domainspecific, hierarchical schema is depicted. The Hierarchical Categorization View divides the Main View into different horizontal slices each of which corresponds to a concept of the hierarchy. Thus, the membership of temporal information depicted in the Main View to the appropriate concept is easy to recognize.

The SemaTime visualization is able to show events and periods, next to (semantically defined) relations between these data elements.

\section{SEMAPROP}

The previous visualizations are designed for presenting a view on the date and its underlying structure. SemaProp has as goal the presentation of only a single data element. In this kind of visualization, detailed information surround this objects will be presented like a list of the properties, relations or pictures and icons.

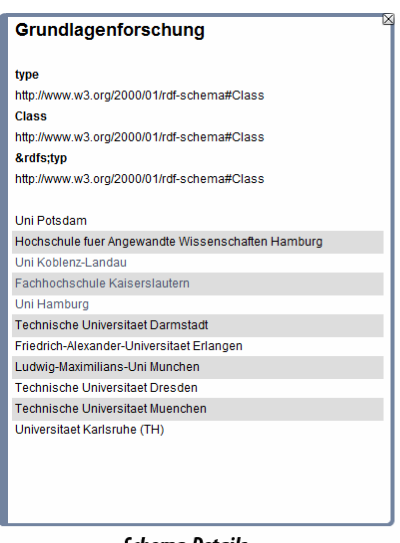

Schema-Details

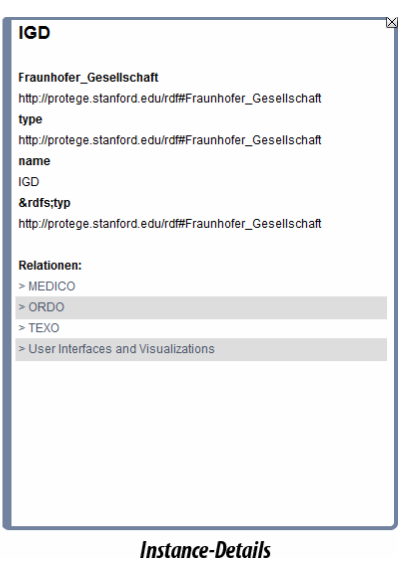

Instance-Details
Figure 6. SemaProp - showing detailed information to a single schema or instance.

A typical use case scenario is the presentation of information to selected instances or concepts within the visualizations from the knowledge cockpit. So the user is able to explore the data and if he founds nodes of interests, he will get further detailed information to it.

\section{Adaptable Composition to a Visualization Cockpit}

The cockpit metaphor is rampant and indicates that different information systems are arranged as a visualization board. The user of a cockpit is always able to see the required information in a predefined area. The information gathering process is very fast and clear, whereas a common cockpit is not interactive and just visualizes only one information unit per cockpit-component. For example a car cockpit visualizes the tempo of the car separately in an own visual interface. It separates the information units from each other and visualizes this information in separate visualization units. The advantage of the separation of complex information units is obvious, the user of a cockpit is able to perceive the required information very fast and react to the perceived information very fast. This kind of information visualization systems has become established in machines and applications, where a fast information perception reaction is necessary, e.g. cars, airplanes etc. Most of these systems do not offer opportunities to interact with them; they are created just for gathering information.
In the field of ontology visualization and exploration a bidirectional communication between the system and the user is given. A proper interaction design within the Visualization Cockpit depends on three attributes: 1. visualization (presentation techniques and metaphors), 2. exploration (linkage, level-of-detail, presentation customization), and 3. ontology (single vs. multiple data sources). While the first attribute had been considered in detail in sections II.B and III.B, the others will be discussed in this chapter.

\section{EXPLORATION}

To support exploration within the ontology the metaphor of the common well established cockpit in e.g. a car will be mapped to the web, to create a system that visualizes a complex information structure. There it is necessary to point out the differences and the main features of such an information system. It is necessary to interact with the Visualization Cockpit. Thus a user can only interact with only one visualization element of the cockpit at a time, the visualization elements should be linked with each other. If a user finds for example a topic of interest and focuses it, all the other visualizations focus this element too. The following figure shows the visualization coupled metaphor, where the same information is shown with two different perspectives. The semaGraph visualization (see Figure 7) just shows the instances of an ontology (right) and the semap (left) visualization shows the hierarchy of the concepts and focuses the related concept.

All the introduced visualizations can be coupled with each other and provide different perspective of the same information in abstracted and different ways as Figure 8 demonstrates. In the figure the visualizations semaSun and semaGraph are linked with each other. The semaSun visualization visualizes the concept hierarchy, multiple inheritances and semantic relations. The information visualization grade is complex and provides more an overview of the focused element, while semaGraph only visualizes the direct relations:

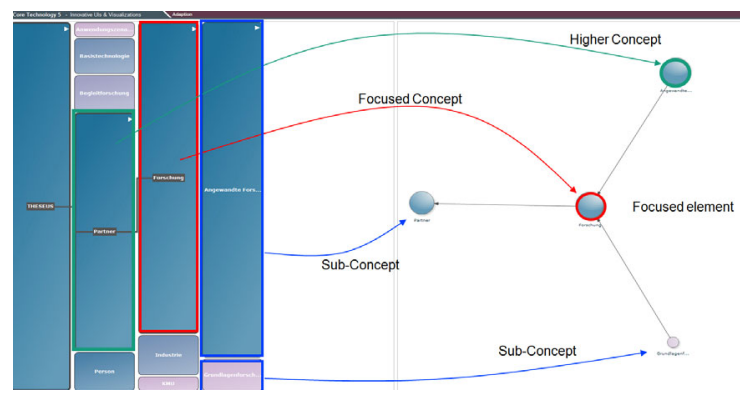

Figure 7. Coupling of two Visualizations

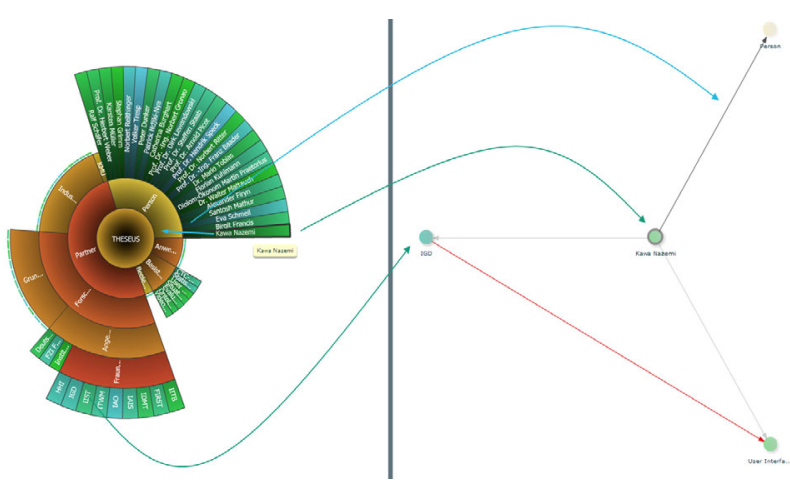

Figure 8. Coupling of SemaSun and SemaGraph 
A visualization Cockpit should provide the possibility to navigate through certain visualizations and the user is able to see the same information in different windows of a user interface. The research on visualization techniques on ontology showed that the complexity of ontology is very difficult to be visualized as just one visualization.

The approach that we developed is the orchestration of different low-level and easy-to-use visualizations for navigation and interaction with ontologies. The user is able to choose the kind of visualization and combine it with other visualizations. He is further able to create a personalized schema for color, order and size of the different visualizations. So he as an individual is able to understand and follow the complex structure of the ontology.

Another main point of the visualization cockpits are the combination of the same visualizations with different zoom levels. The zoom levels may vary from visual zoom, to semantic zoom with semantics based filtering. For example the level of detail can on the hand be used to show a greater part of the ontology or information space for showing the structure of the information and on the other hand with small numbers of elements of interest to show detailed information. There are two main ways to combine the same visualization technique duplicated in a cockpit for providing more information. First the level of details can be provided as a zoom on a specific area of the ontology versus the whole search results. Figure 9 shows the same visualizations semaGraph with different levels of visual zoom for providing different levels of information, whereas the context is not lost and the user or learner is able to recognize which part of the ontology is on focus:

Another method for visualizing the same information with the same visualization and different parameters is using different numbers of entities. A reduction of the numbers of entities can be achieved by semantically filtering the information, e.g. based on relevance metric, or by chunking information, e.g. based on clustering. With this kind of information visualization a similar effect can be achieved. Many information elements gives an overview about the whole structure of the data and ontology respectively and the information about the focused element can be revealed with a visualization which visualizes a small number of elements. The following figure shows the second variant of using the same information and same visualization to visualize different aspects:

Using orchestrated visualizations for exploring information spaces another important aspect for the user are visual cues to identify similar information items. This is crucial to establish and retain the awareness which part of the information space is actually visualized. For example most systems having an overview window display a colored rectangle within this overview indicating the boundary of the detailed view. With an increasing difference in the zoom levels of these two visualizations the probability of a 'lost in (information) space' for the user raises.

To achieve this awareness other visual essentials may be used. User studies revealed that some of these visual essentials are valuable whereas others are misleading for the user because they associated another meaning underlying. For example the visual essentials color and shape are well suited for retaining informational awareness. The essential color obtained the best reliability in our user studies, therefore this essential is used in all figures in this

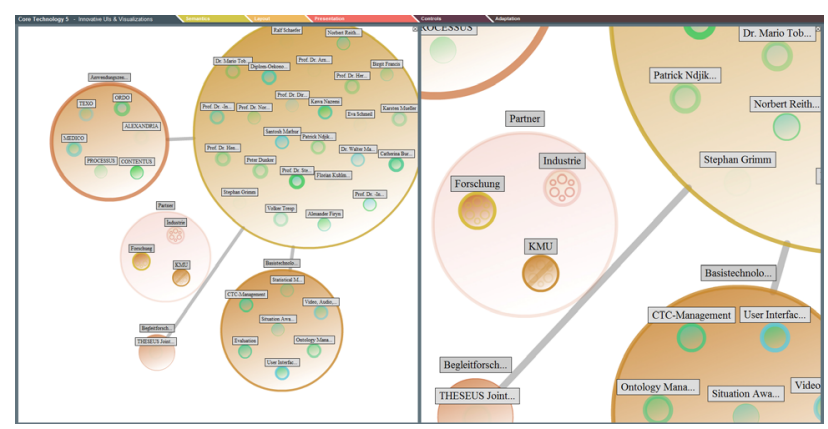

Figure 9. Level-of-Details by Zoom

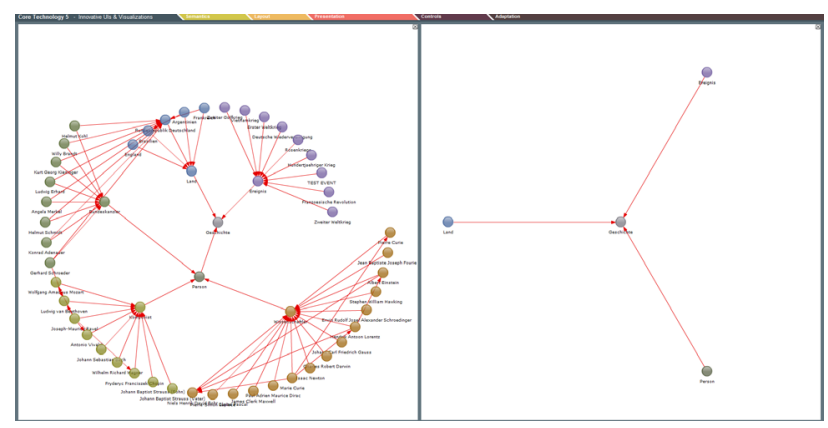

Figure 10. Level-of Details by Amount of Elements

work to declare similar information items. In Figure 7 the essential space is used in addition.

In contrast to these positive examples the order had not been interpreted correct, except for the visualization of hierarchically structured information. Another useful visual essential is the border style of the elements. But border styles can only be used for a limited number of disjoint elements because the visual scope of distinguishable styles is limited, for example different thicknesses and colors for solid, dashed and dotted styles. Therefore the border style is more applicable for distinguishing clusters or sets of similar information elements.

Besides the visual cues linking different visualizations on an informational or respectively semantic manner, the linkage of visualizations on the interaction level is a helpful feature for exploring information and thus imparting knowledge for the user. We identified two useful interaction linkages: none and focus. A third possible interaction linkage is highlighting similar information items in different visualizations when the user moves the mouse over or selects one item. But in this context highlighting is more helpful when it is used as a visual essential to achieve informational awareness, discussed before.

The adequate interactional linkage depends on the users' intention and his task he is working on. For example linking the focus (e.g. actual focussed information element is positions in the views' center) in different visualizations is suitable when different zoom levels are used, like Figure 10 illustrates for semantic zoom. In the overview metaphor an interaction linkage is used for the visual zoom, as far as a rectangle is used to indicate the boundaries of the detailed view.

No interactional linkage may be useful for the user if he wants to compare two or more different excerpts of the information space. For example an analyst wants to identify and analyse the impact factors for different companies' stock markets. 
Likewise unlinked visualizations are used for the visualization of different information spaces, respectively information sources. This leads to the third attribute Ontology which is discussed in the next subsection.

\section{ONTOLOGY}

The combination of different ontologies from multiple information sources became more and more important in recent times. For example with the growing trend for linking different data sources like it is done in the field of linked open data, also the need for visualizing different ontologies arise. Especially the process of evaluating links between open data to maintain quality assurances (or to ensure service level agreements in an economically context) can be assisted by the orchestration of visualizations of these data, where each data source may again be visualized with multiple visualizations for the reasons discussed above.

This orchestration of visualizations presenting different ontologies determines possibilities and restrictions of the other attributes for visualization orchestration, especially the Exploration. In detail visualizations of different ontologies are often unlinked (in the interactional manner) which can primarily be put down to the fact that automatic approaches for identifying information items with the same meaning are not dependable enough, till now.

Technical restrictions (in an algorithmic meaning) limit the ability to automatically identify similar or mostly equally information items in different sources. The referenced meaning may be different even if the items are identified by the same id or similarly labeled. For example synonyms have similar labels, e.g. 'bush' can have at least four different meanings. Using ontologies this problem is reduced for the comparison of ids, because one goal of the Semantic Web is the unique identification of real world resources [28]. But even here this is no reliable indication, e.g. when the information is gathered automatic and signed with artificial ids. Another reason can be the individually and contextually modeled ontologies, for example for a person named 'John Smith' in different sources.

Nevertheless the unlinked orchestration of different ontologies is beneficial for the user interacting and exploring these data sources. If the user wants to e.g. gather detailed information about a politician he may want to combine different data sources. In this example one data source may contain time-dependent information about the political CV. Another data source may point out detailed information about his or her campaign, associated slogans and published reports. A third data source may provide media information like pictures and videos. By this orchestration the user can easily navigate in one visualization to extract specific information items, which he can visually bring in relation to other extracted information items (of the same or of another data source). Thus he can easily achieve a comprehensive impression of this politician.

The cockpit metaphor combines different aspects of visualization with each other and provides different views and perspectives respectively: 1) Visualization of the same data using the same visualizations with different parameters (Level-of-Details). 2) Visualization of the same data with different linked visualization-types (perspectives). 3. Visualization of different data with the same visualizationtypes (comparison of information.). 4) Visualization of different data with different visualization-types (aspect- oriented visualization). With the different adjustments of the visualization cockpits, different goals of a learner or information searcher can be achieved and to different requirements can be responded.

\section{IMPLEMENTATION}

To provide the functionalities described in section III our implemented system of the visualization cockpit is developed highly modular. Different visualizations realize various presentation techniques and metaphors. For the reason of loosely coupling all the visualizations were developed with the same functionalities (presentation and level-of-detail customization) and are sending messages in the background about user interaction events. These messages are handled within the framework and are forwarded to destinated visualizations, thus to linked visualizations. Furthermore the framework offers the ability to load different data sources which can be loaded in a visualization the user specifies.

The user starts with opening a single visualization and can add more visualization to create his own Knowledge Cockpit. Further he is able to personalize and adapt all the graphical presentation elements, like color, complexity, hierarchy etc.

The following figure shows different combinations of Knowledge Cockpits created by users:

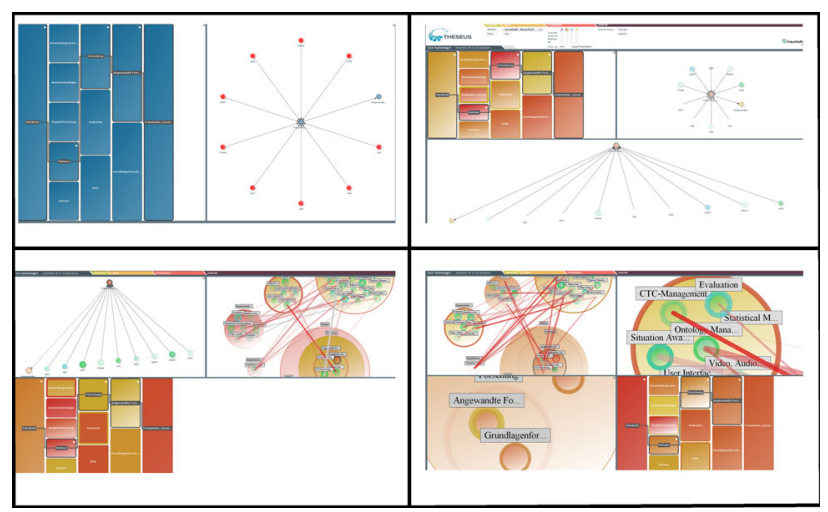

Figure 11. Different Visualization Cockpits

\section{CONCLUSION}

Nowadays information are stored in large digital libraries, like semantic databases in kind of ontologies, which allowing many modern strategies for searching information. But because of the size, most of the traditionally search strategies and also the form for presenting the information to the users are not so effective anymore. New approaches are necessary to allow the user an individual view on these large datasets, a view which show him only that kind of information, which he currently wanted to know. An important aspect is the type of information visualization and also, how the user is able interact through this data, because in case of difficult data exploration, it will overstrain the user. So the intuitiveness of information visualization is a central aspect for supporting the users by his work with large datasets. In consequence the users must be able to concentrate on their main task and not in learning to use the application. Another challenge by using such complex semantic databases is the understanding, what information the user is searching for 
and even more, what kind of information have to be presented.

In this paper we presented a strategy to support the user by exploring especially large semantically knowledge bases. The main aspects lay on the so called "Knowledge Cockpit", where the users are able to orchestrate their own cockpit, which will fits best to user's goal of searching information. For the cockpit, the user will have a set of different aspect oriented visualization, which the user can use and combine with each other, so that the navigation through the data is individualized to the requirements of the user. The visualization support thereby different kinds of aspects, for instance the SeMap is primary designed for a fast navigation through the schema-level, in difference to the SemaGraph that is useful for showing only direct related elements up to a defined level-of-detail. In difference to these kinds of aspect oriented visualization, a SemaTime is only useful for time annotated data, but it is powerful by analyzing chronological data like events or periods. So the user gets a powerful tool, where he is able to select those kinds of information visualization that will support his exploration or searching process. Basing on these aspects, we can summarize that a proper interaction design within the Visualization Cockpit depends on three characteristics:

1. The support of different kinds of visualization and its adaptability of the presentation, which subsumes the support of different presentation techniques and presentation metaphors.

2. The possibility for a data exploration. To regard that fact, the datasets must contain linkage between different instances. Also the user must be able to set the level-of-detail and to customize the presentation to his personal perceptions.

3. The selection of semantic databases, so that single or multiple data sources can be presented in the cockpit. In combination of different data source, the user can compare the contents within his knowledge cockpit.

The first characteristic had been considered in detail in sections II.B and III.B. The second characteristic is a general but also important aspect. Because data exploration is only possible, if related instances are linked with each other, so that a user gets the possibility to navigate within a graphical visualization along the edges. If no relations are integrated, a user has no intuitive possibility the get further topic related information. The third characteristic encompasses the flexible use of the knowledge cockpit and also its concrete use cases. Similar to a traditional investigation a user will be able to compare different data sources or parts of it. So on the one hand coupled visualization helps to navigate through a dataset and showing the same features in different ways in different selected visualizations. On the other hand the user needs the possibility to compare parts of the database or rather to compare different data sources with each other. So the visualization must also be uncoupled.

With the presented approach for a knowledge cockpit the user will have a powerful tool to explore large semantics databases on an intuitive way. The cockpit consists only of aspect oriented visualization, which the user suggests as helpful and intuitive. So the user can focus on his tasks for searching and exploring information.

\section{REFERENCES}

[1] Alani, H.: TGVizTab: An Ontology Visualization Extension for Protégé Knowledge Capture (K-Cap’03), Workshop on Visualization Information in Knowledge Engineering (2003)

[2] Artino, A.R.Jr.: Cognitive Load Theory and the Role of Learner Experience: An Abbreviated Review for Educational Practitioners. In: AACE Journal, vol. 16 (4), pp. 425--439. (2008)

[3] Baehrecke, E., Dang, N., Babaria, K. and Shneiderman, B.: Visualization and analysis of microarray and gene ontology data with treemaps. BMC Bioinformatics (5), pp. 1--12 (2004)

[4] Bhatti, N.: Web Based Semantic Visualization to Explore Knowledge Spaces - An Approach for Learning by Exploring. Proceedings of ED-Media 2008, pp. 312--317 (2008)

[5] Bosca, A.; Bonino, D., Pellegrino, P.: OntoSphere: more than a 3D ontology visualization tool. Proceedings of SWAP 2005, the 2nd Italian Semantic Web Workshop, CEUR Workshop Proceedings (2005)

[6] Carol, J.J., Klyne, G.: Resource Description Framework: Concepts and Abstract Syntax. W3C Recommendation (2004). http://www.w3.org/TR/2004/REC-rdf-concepts-20040210/

[7] Eklund, P., Roberts, N., Green, S.,: OntoRama: Browsing RDF ontologies using a hyperbolic-style browser. Cyber Worlds, 2002. Proceedings Frist International Symposium on, pp. 405--411 (2002)

[8] Federal Ministry of Economy and Technology, Bundesministerium für Wirtschaft und Technologie (BMWi), http://www.bmwi.de/

[9] Fitts, P.M.: The Information Capacity of the Human Motor System in Controlling the Amlitude of Movement. In: Journal of Experimental Psychology, vol. 47, pp. 381--391. Psychology Press (1954)

[10] Freire, M., Rodríguez, P.: Preserving the mental map in interactive graph interfaces. In: Proceedings of Advanced Visual Interfaces. ACM, New York (2006)

[11] Gruber, T.: A Translation Approach to Portable Ontology Specifications. Knowledge Acquisition, Vol. 5, Issue 2, pp. 199-220 (1993) doi:10.1006/knac.1993.1008

[12] Herman, I., Melancon, G., Marshall, M.S.: Graph Visualization and Navigation in Information Visualization: A Survey. In: IEEE Transactions on Visualization and Computer Graphics, vol. 6 (1), pp. 24--43. IEEE (2000)

[13] Katifori, A., Halatsis, C. Lepouras, G. Vassilakis, C., Giannopoulou, E.: Ontology Visualzation Methods - A Survey. In: ACM Computing Surveys, vol. 39 (4). ACM, New York (2007)

[14] Kerrigan M., WSMOViz: An Ontology Visualization Approach for WSMN. Information Visualization. IV 2006 Tenth International Conference on, pp. 411--416 (2006)

[15] Krigelstein, S., Motschnig-Pitrik, R.: Knoocks: New Visualization Approach for Ontologies. Proceedings of the 2008 12th International Conference Information Visualization, IEEE Computer Society (2008)

[16] Liebig, T., Noppens, O.: Onto Track: Combining Browsing and Editing with Reasoning and Explaining for OWL Lite Ontologies. Proceedings of the 3rd International Semantic Web Conference (ISWC2004), Springer, pp. 244--257 (2004)

[17] Nazemi, K., Breyer, M., Hornung, C.: SeMap: A Concept for the Visualization of Semantics as Maps UAHCI '09: Proceedings of the 5th International Conference on Universal Access in HumanComputer Interaction. Part III, Springer, pp. 83--91 (2009)

[18] Noy, N., F., Fergerson, R., W., Musen, M., A.: The Knowledge Model of Protégé-2000: Combining Interoperability and Flexibility. EKAW '00: Proceedings of the 12th European Workshop on Knowledge Acquisition, Modeling and Management, Springer pp. 17--32 (2000)

[19] Paraskakis, I.: Ambient Learning: a new paradigm for e-learning. In: 3rd International Conference on Multimedia and Information \& Communication Technologies in Education (2005)

[20] Parsia, B., Wang, T., Golbeck, J.: Visualizing web Ontologies with CropCircles. End User Semantic Web Interactions WS @ ISWC2005 (2005) 
[21] Pietringa, E.: IsaViz: A Visual Authoring Tool for RDF. http://www.w3.org/2011/11/IsaViz (2004)

[22] Plaisant, C., Grosjean, J., and Bederson, B. (2002). SpaceTree: supporting exploration in large node link tree, design evolution and empirical evaluation. Information Visualization. INFOVIS 2002. IEEE Symposium, 57-64

[23] The Personalization Consortium, http://www.personalization.org/ personalization.html

[24] Shneiderman, B.: The Eyes Have It: A Task by Data Type Taxonomy for Information Visualizations. In: Proceedings 1996 IEEE, Visual Languages, pp. 336--343. IEEE, Washington (1996)

[25] Shneiderman, B.: Tree Visualization with Tree-Maps: 2-d SpaceFilling Approach. In: ACM Transactions on Graphics, vol. 11 (1), pp. 92--99. ACM, New York (1992)

[26] Sintek, M.: OntoViz tab: Visualizing Progégé ontologies. http://protegewiki.stanford.edu/index.php/OntoViz (2003)

[27] Storey, M., Musen, M., Silva. J., Best, C., Ernst, N., Fergerson, R., Noy, N.: Jambalaya: Interactive visualization to enhance ontology authoring and knowledge acquisition in Protégé. Workshop on Interactive Tools for Knowledge Capture (K-CAP-2001) (2001)

[28] Studer, R., Davies, J., Warren, P.: Semantic Web Technologies Trends and Research in Ontology-Based Systems. John Wiley \& Sons. (2006)

[29] THESEUS Program, http://www.theseus-programm.de

[30] UDLA Universidad de las Americas Puebla, Image Source: http://ict.udlap.mx/people/carlos/is215/images/visualization/

[31] VanHam, F., van Wijk, J.: Beamtrees: compact visualization of large hierarchies. Information Visualization, 2002. INFOVIS 2002. IEEE Symposium on, pp. 93--100 (2002)

[32] W3C RDF Specification, http://www.w3.org/RDF/

[33] Ware, C.: Information Visualization: Perception for Design. Morgan Kaufmann, San Francisco (2000)

[34] World Wide Web Consortium, http://www.w3.org/

[35] Stab, C; Breyer, M.; Nazemi, K.; Burkhardt, D.; Hofmann, C.; Fellner, D. W.: SemaSun: Visualization of Semantic Knowledge based on an improved Sunburst Visualization Metaphor. In: Association for the Advancement of Computing in Education (AACE): Proceedings of ED-Media 2010: World Conference on Educational Multimedia, Hypermedia \& Telecommunications. Toronto (2010).

[36] Stab, C.; Nazemi, K.; Fellner, D.W.: SemaTime - Timeline Visualization of Time-Dependent Relations and Semantics. In Proceedings of the 6th International Symposium on Visual Computing (ISVC 2010), Las Vegas (2010).

\section{AUTHORS}

K. Nazemi is senior research scientist at the Fraunhofer Institute for Computer Graphics Research in Darmstadt, Germany and holds courses at the Technical University Darmstadt. His research interests focuses on user-, dataand context adaptive information and knowledge visualizations (email: kawa.nazemi@igd.fraunhofer.de).

M. Breyer is employed as a research scientist at the Fraunhofer Institute for Computer Graphics Research, Darmstadt Germany. His research focus is the analysis of processes for semantic processing of heterogeneous data and data structures for the purpose of visualizing them and the data driven optimization of presentation and interaction techniques within these visualizations. (e-mail: matthias.breyer@igd.fraunhofer.de)

D. Burkhardt is employed as a research scientist at the Fraunhofer Institute for Computer Graphics Research, Darmstadt Germany. His research interests are focused on technologies and concepts for process support of domainexpert during semantic editing and annotation processes. He also researches in gesture-based multimodal interactions. (e-mail: dirk.burkhardt@igd.fraunhofer.de)

D. W. Fellner is professor of computer science at Technical University Darmstadt, Germany, and director of the Fraunhofer Institute for Computer Graphics Research (IGD) at the same location. In the areas of computer graphics and digital libraries he is a member of the editorial boards of leading journals and a member of the program committees of many international conferences and workshops. He was also program co-chair of the Eurographics'97 and Eurographics'2003 conference. In 2008 and 2009 he was program co-chair and conference cochair of the ACM SIGGRAPH Web3D conference. (email: institutsleitung@igd.fraunhofer.de).

This work was supported in part by the German Federal Ministry of Economics and Technology as part of the THESEUS Research Program. For more information please see http://www.semavis.com.

Submitted October $10^{\text {th }}, 2010$. Published as resubmitted by the authors October $17^{\text {th }}, 2010$. 